02

\title{
Эффект близости в сверхпроводящем триплетном спиновом клапане S1/F1/S2/F2
}

\author{
(C) Р.Р. Гайфуллин ${ }^{1}$, В.Н. Кушнир ${ }^{2,3}$, Р.Г. Деминов ${ }^{1}$, Л.Р. Тагиров ${ }^{1,4}$, М.Ю. Куприянов ${ }^{1,5,6}$, А.А. Голубов ${ }^{6,7}$ \\ ${ }^{1}$ Институт фризики Казанского (Приволжского) федерального университета, \\ Казань, Россия \\ ${ }^{2}$ Белорусский государственный университет информатики и радиоэлектроники, \\ Минск, Беларусь \\ ${ }^{3}$ Белорусский государственный университет, \\ Минск, Беларусь \\ ${ }^{4}$ Казанский фризико-технический институт им. Е.К. Завойского ФИЦ Казанский научный центр РАН, \\ Казань, Россия \\ ${ }^{5}$ Научно-исследовательский институт ядерной физики им. Д.В. Скобельцына \\ Московского государственного университета, \\ Москва, Россия \\ ${ }^{6}$ Московский фризико-технический институт, \\ Долгопрудный, Россия \\ ${ }^{7}$ Faculty of Science and Technology and MESA + Institute of Nanotechnology University of Twente, \\ Enschede, The Netherlands \\ E-mail: gaifullin.rashid@gmail.com
}

Поступила в Редакцию 15 апреля 2019 г.

В окончательной редакции 22 апреля 2019 г.

Принята к публикации 24 апреля 2019 г.

С помощью матричного метода решения линеаризованных уравнений Узаделя получены критические температуры многослойных структур вида сверхпроводник/ ферромагнетик/ ферромагнетик (S/F/F). Pacсмотрено влияние дополнительного слоя сверхпроводника на эффекты трехслойного спинового вентиля. В сравнении с дополнительным нормальным слоем в структуре $\mathrm{S} / \mathrm{F} / \mathrm{N} / \mathrm{F}$, обсуждена возможность увеличения эффективности режимов спинового вентиля с дополнительным сверхпроводящим слоем $\mathrm{S}$ на месте слоя $\mathrm{N}$.

Ключевые слова: сверхпроводимость, ферромагнетизм, эффект близости, критическая температура, спиновый вентиль.

DOI: $10.21883 /$ FTT.2019.09.48093.21N

\section{1. Введение}

Взаимное влияние на границе раздела двух конкурирующих состояний - сверхпроводимости и ферромагнетизма - является предметом интенсивных экспериментальных и теоретических исследований в течение последних двух десятилетий. Одно из самых удивительных проявлений взаимодействия между ними - немонотонная зависимость критической температуры перехода в сверхпроводящее состояние, $T_{\mathrm{c}}$, от толщины ферромагнитного слоя в системе сверхпроводник / ферромагнетик. Ранее было показано, что $T_{\mathrm{c}}$ трехслойной структуры $\mathrm{S} / \mathrm{F} 1 / \mathrm{F} 2$ [1] (S - синглетный сверхпроводник, F1 и F2 ферромагнитные металлы) и многослойной структуры $\mathrm{S} / \mathrm{F} 1 / \mathrm{N} / \mathrm{F} 2$ [2] (N - нормальный металл) может быть немонотонной функцией угла $\alpha$ между намагниченностями двух ферромагнитных слоев, в противоположность монотонному поведению $T_{\mathrm{c}}(\alpha)$, полученному для трехслойной гетероструктуры F1/S/F2 [3]. Это свойство может быть использовано для переключения гетероструктуры из сверхпроводящего состояния в нормальное и обратно, прикладывая небольшое магнитное поле в определенном направлении [1,2], то есть изготовления сверхпроводящего спинового клапана, обладающего бесконечным магнетосопротивлением. Имеется ряд экспериментальных работ (см., например, цитирования в обзоре [4]), в которых наблюдалась зависимость $T_{\mathrm{c}}$ от угла между магнитными моментами в $\mathrm{S} / \mathrm{F} 1 / \mathrm{N} / \mathrm{F} 2$ структуре с одинаковыми ферромагнетиками в качестве F слоев. Максимальная разность температур оказалась в пределах $50 \mathrm{mK}$, поэтому для увеличения эффекта один из металлических ферромагнетиков группы железа (F2) был заменен на ферромагнитный полуметаллический оксид хрома $\mathrm{CrO}_{2}$ [5] или на ферромагнитный полуметаллический сплав Хойслера [6]. В настоящей работе рассмотрено влияние дополнительного сверхпроводящего слоя S2 в гетероструктуре S1/F1/S2/F2, в которой дальнодействующая триплетная компонента сверхпроводящего спаривания генерируется при неколлинеарной ориентации намагниченностей ферромагнитных слоев [7]. Рассчитана зависимость $T_{\mathrm{c}}(\alpha)$ от толщины дополнительного сверхпроводящего слоя S2. В сравнении со структурой S/F1/N/F2 обсуждается, который из слоев и каким образом влияет на режимы спинового 
клапана. Исследованы условия, при которых сверхпроводимость в дополнительном слое S2 подавляется, и он выполняет функции нормального слоя, и условия, когда сверхпроводимость этого слоя сохраняется и влияет на температуру перехода $T_{\mathrm{c}}$ гетероструктуры.

\section{2. Модель и численный метод}

Рассматривается бесконечная в направлениях $у$ и $z$ структура $\mathrm{S} 1 / \mathrm{F} 1 / \mathrm{S} 2 / \mathrm{F} 2$ (рис. 1). Обменное поле $F 1$ слоя находится в $y z$ плоскости, $\mathbf{h}=(0, h \sin \alpha, h \cos \alpha)$, тогда как обменное поле $F 2$ слоя направлено вдоль оси $z$, $h=(0,0, h)$. Угол $\alpha$ меняется от 0 (параллельная конфигурация, П) до $\pi$ (антипараллельная конфигурация, АП).

Для вычисления критической температуры $T_{\mathrm{c}}$ как функции параметров спинового клапана, который в диффузионном пределе описываться с помощью уравнений Узаделя, был применен матричный метод [8-10].

При моделировании использовалось следующие приближения: все границы контактов прозрачны $\left(\gamma_{\mathrm{B}}=0\right)$, константы диффузии и удельные сопротивления одинаковы $(\gamma=1)$, абсолютные значения обменных полей в обоих ферромагнитных слоях совпадают.

\section{3. Результаты и обсуждение}

Результаты численных расчетов $T_{\mathrm{c}}$ как функции угла $\alpha$ и толщин S2 и $\mathrm{N}$ слоев гетероструктур S1/F1/S2/F2 и $\mathrm{S} 1 / \mathrm{F} 1 / \mathrm{N} / \mathrm{F} 2$ представлены на рис. 2-5.

На рис. 2 представлен прямой режим переключения спинового вентиля $\left(T_{\mathrm{c}}^{\mathrm{A} \Pi}\left(\alpha=180^{\circ}\right)>T_{\mathrm{c}}^{\Pi}\left(\alpha=0^{\circ}\right)\right)$, который реализуется при тонких ферромагнитных слоях.

На рис. 3 представлен триплетный режим спинового вентиля $\left(T_{\mathrm{c}}\right.$ (неколлинеарная) $\left.)<T_{\mathrm{c}}^{\Pi}, T_{\mathrm{c}}^{\mathrm{A}}\right)$, который реализуется при немного больших толщинах ферромагнитных слоев.

На рис. 4 представлена критическая температура $T_{\mathrm{c}}$ для параллельной и антипараллельной ориентации намагниченностей как функции толщины дополнительных $\mathrm{S}$ и $\mathrm{N}$ слоев для прямого режима переключения.

На рис. 5 представлена критическая температура $T_{\mathrm{c}}$ для параллельной и ортогональной ориентации намагниченностей как функции толщины дополнительных $\mathrm{S}$ и $\mathrm{N}$ слоев для триплетного режима переключения.

На рис. 6 представлена разница между критическими температурами $\Delta T_{\mathrm{c} d i r}=T_{\mathrm{c}}^{\mathrm{A \Pi}}-T_{\mathrm{c}}^{\Pi}$ для антипараллельной и параллельной ориентации намагниченностей как функция толщины дополнительных $\mathrm{S}$ и $\mathrm{N}$ слоев для прямого режима переключения.

На рис. 7 представлена разница между критическими температурами $\Delta T_{\mathrm{c} \text { tr }}=T_{\mathrm{c}}^{\Pi}-T_{\mathrm{c}}\left(\alpha=90^{\circ}\right)$ для параллельной и ортогональной ориентации намагниченностей как функция толщины дополнительных $\mathrm{S}$ и $\mathrm{N}$ слоев для триплетного режима переключения.

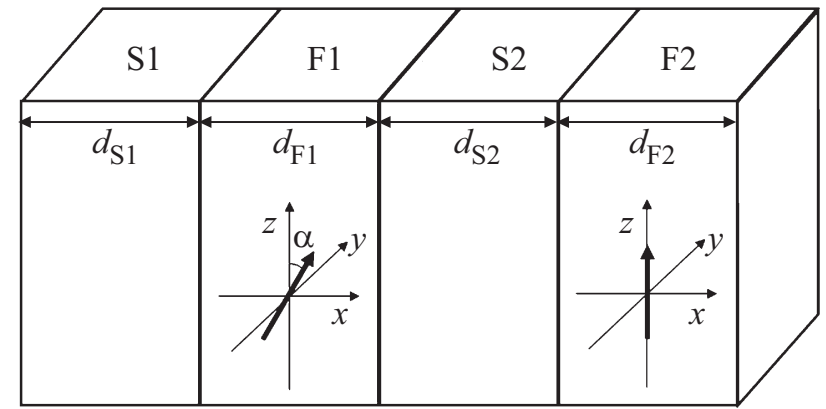

Рис. 1. Гетероструктура S1/F1/S2/F2. Внешняя граница слоя S1 соответствует координате $x=0$. Жирные стрелки в $\mathrm{F}$ слоях обозначают направления обменных полей $\mathbf{h}$, лежащих в $y z$ плоскости.

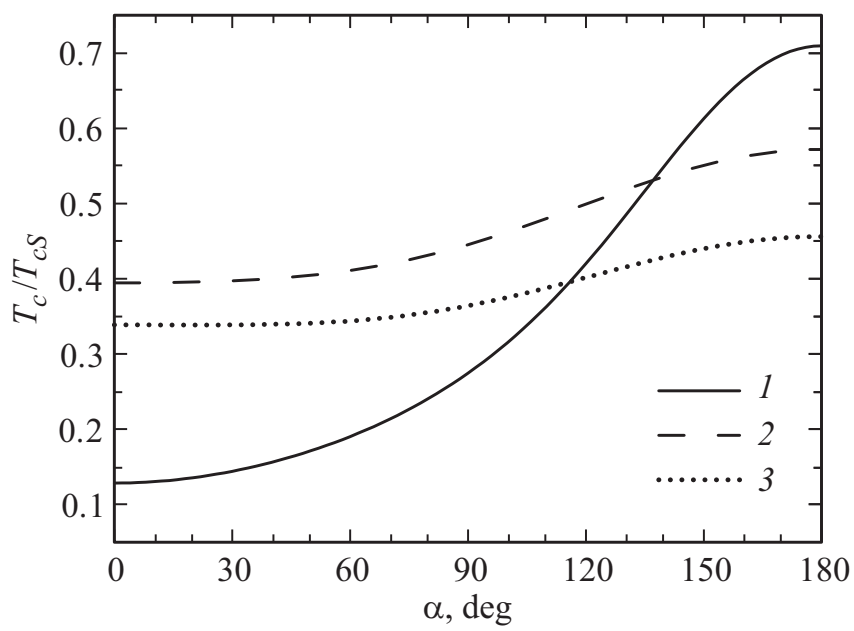

Рис. 2. Критическая температура $T_{\mathrm{c}}$ в зависимости от угла $\alpha$ для структуры S1/F1/F2 (1), S1/F1/S2/F2 (2), S1/F1/N/F2 (3). Толщины слоев $d_{\mathrm{S} 1} / \xi_{\mathrm{S} 1}=2.76, d_{\mathrm{F} 1} / \xi_{\mathrm{F} 1}=0.15, d_{\mathrm{F} 2} / \xi_{\mathrm{F} 2}=0.15$, $d_{\mathrm{S} 2} / \xi_{\mathrm{S} 2}=d_{\mathrm{N}} / \xi_{\mathrm{N}}=1$, режим прямого переключения.

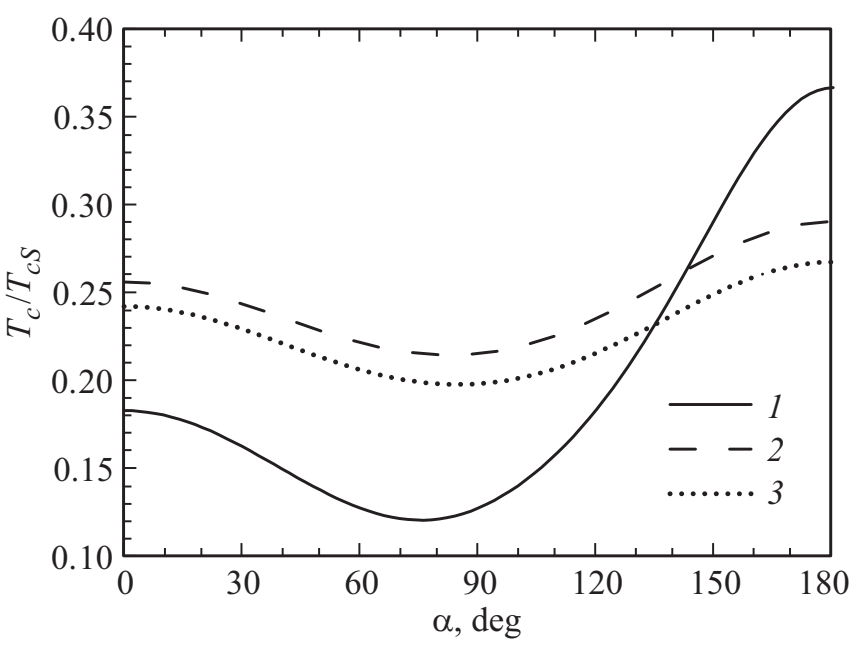

Рис. 3. Критическая температура $T_{\mathrm{c}}$ в зависимости от угла $\alpha$ для структуры S1/F1/F2 (1), S1/F1/S2/F2 (2), S1/F1/N/F2 (3). Толщины слоев $d_{\mathrm{S} 1} / \xi_{\mathrm{S} 1}=2.76, d_{\mathrm{F} 1} / \xi_{\mathrm{F} 1}=0.3, d_{\mathrm{F} 2} / \xi_{\mathrm{F} 2}=0.7$, $d_{\mathrm{S} 2} / \xi_{\mathrm{S} 2}=d_{\mathrm{N}} / \xi_{\mathrm{N}}=1$, режим триплетного переключения. 
Для прямого режима спинового клапана со сверхпроводящим слоем среднее значение температуры увеличивается, тогда как с нормальным слоем оно практически не изменяется, рис. 4. Из рис. 6 видно, что увеличение толщины дополнительного слоя уменьшает амплитуду изменения температуры. В сравнении с нормальным слоем, сверхпроводящий слой увеличивает эффективность прямого режима, больше становятся как амплитуда, так и среднее значение изменения $T_{\mathrm{c}}$.

Для триплетного режима со сверхпроводящим слоем среднее значение температуры увеличивается, так же, как и в прямом режиме, рис. 5. Из рис. 7 видно, что при небольшой толщине дополнительного слоя амплитуда изменения температуры даже незначительно увеличива-

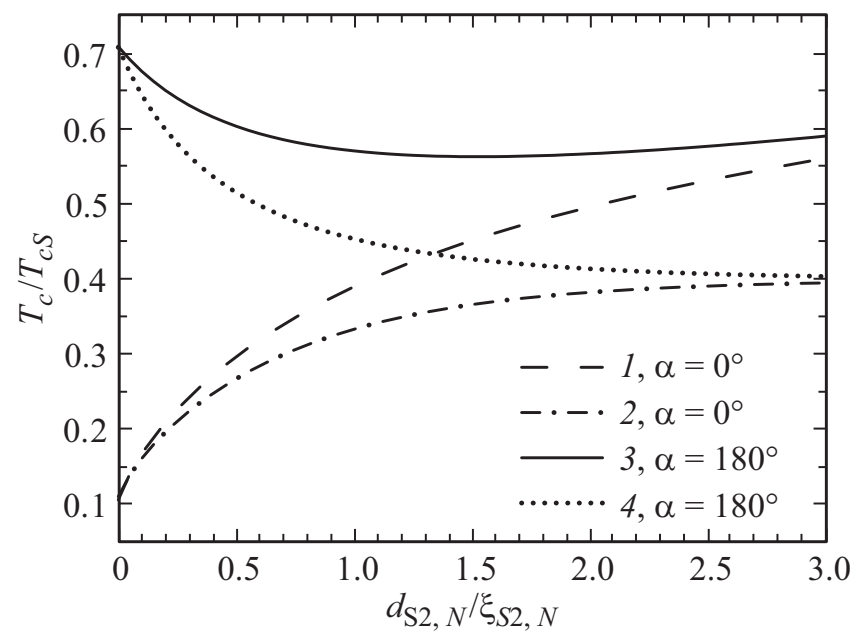

Рис. 4. Зависимость критической температуры $T_{\mathrm{c}}$ от толщины $d_{\mathrm{S} 2}$ структуры $\mathrm{S} 1 / \mathrm{F} 1 / \mathrm{S} 2 / \mathrm{F} 2(1,3)$ и от толщины $d_{\mathrm{N}}$ структуры $\mathrm{S} 1 / \mathrm{F} 1 / \mathrm{N} / \mathrm{F} 2(2,4)$. Толщины других слоев $d_{\mathrm{S} 1} / \xi_{\mathrm{S} 1}=2.75$, $d_{\mathrm{F} 1} / \xi_{\mathrm{F} 1}=0.15, d_{\mathrm{F} 2} / \xi_{\mathrm{F} 2}=0.15$.

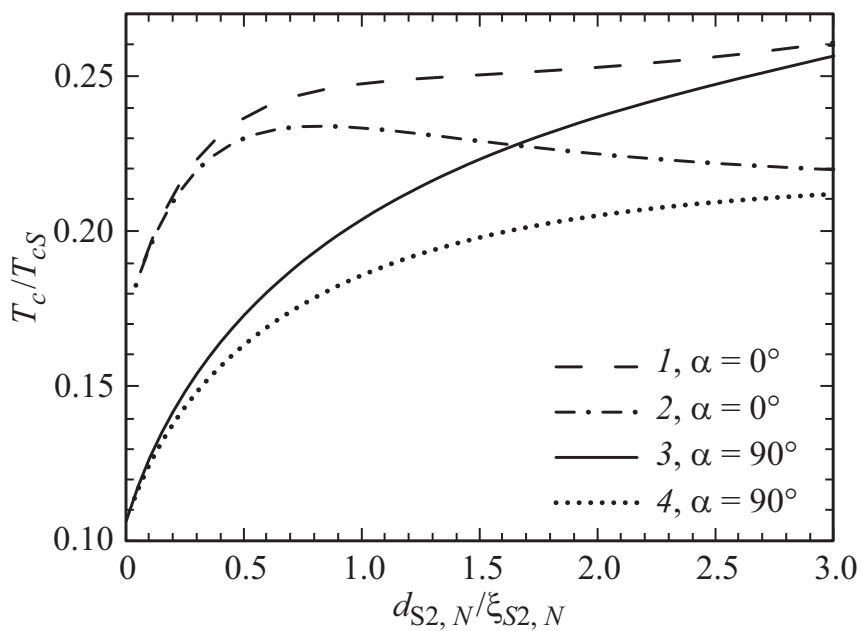

Рис. 5. Зависимость критической температуры $T_{\mathrm{c}}$ от толщины $d_{\mathrm{S} 2}$ структуры $\mathrm{S} 1 / \mathrm{F} 1 / \mathrm{S} 2 / \mathrm{F} 2(1,3)$ и от толщины $d_{\mathrm{N}}$ структуры $\mathrm{S} 1 / \mathrm{F} 1 / \mathrm{N} / \mathrm{F} 2(2,4)$. Толщины других слоев $d_{\mathrm{S} 1} / \xi_{\mathrm{S} 1}=2.75$, $d_{\mathrm{F} 1} / \xi_{\mathrm{F} 1}=0.3, d_{\mathrm{F} 2} / \xi_{\mathrm{F} 2}=0.7$.

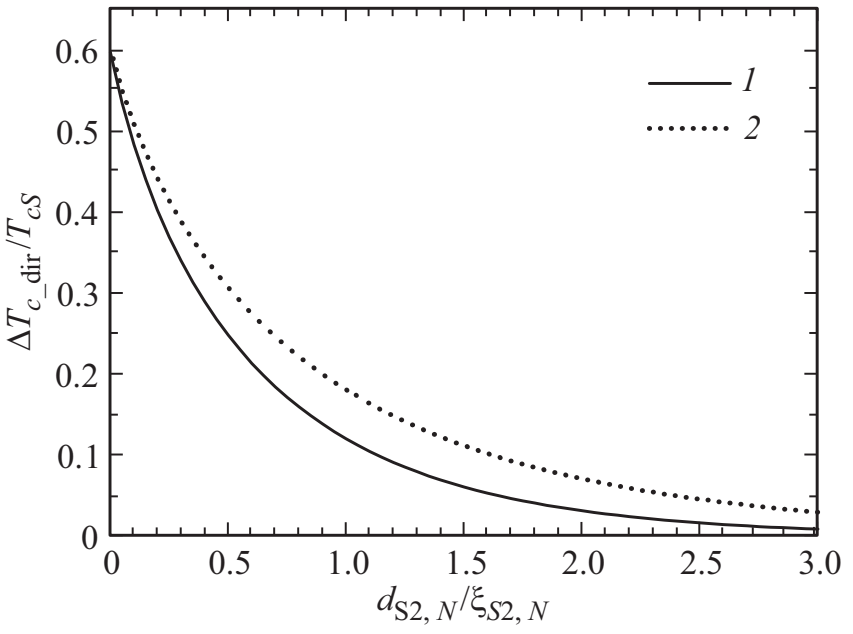

Рис. 6. Зависимость $\Delta T_{\mathrm{c} \text { dir }}$ от толщины $d_{\mathrm{N}}$ структуры $\mathrm{S} 1 / \mathrm{F} 1 / \mathrm{N} / \mathrm{F} 2$ (1) и от толщины $d_{\mathrm{S} 2}$ структуры S1/F1/S2/F2 (2) для прямого режима.

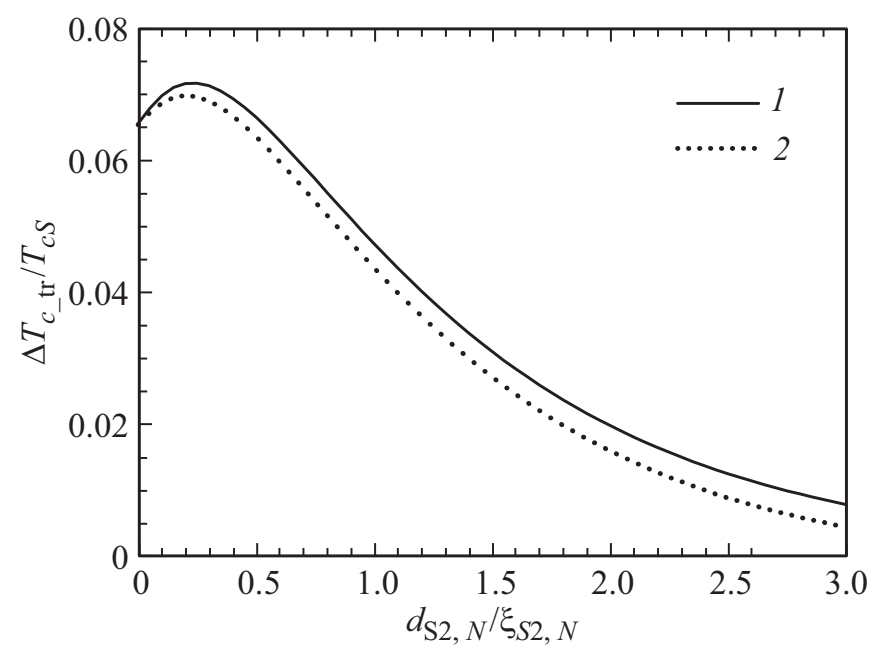

Рис. 7. Зависимость $\Delta T_{\mathrm{c} \text { tr }}$ от толщины $d_{\mathrm{N}}$ структуры $\mathrm{S} 1 / \mathrm{F} 1 / \mathrm{N} / \mathrm{F} 2$ (1) и от толщины $d_{\mathrm{S} 2}$ структуры $\mathrm{S} 1 / \mathrm{F} 1 / \mathrm{S} 2 / \mathrm{F} 2$ (2) для триплетного режима.

ется. Амплитуды изменения температуры S1/F1/S2/F2 и S1/F1/N/F2 структур примерно равны.

При больших толщинах ферромагнитных слоев, при которых реализуется инверсный режим спинового клапана $\left(T_{\mathrm{c}}^{\mathrm{A} \Pi}<T_{\mathrm{c}}^{\Pi}\right)$, сверхпроводящее состояние в $S 2$ слое подавляется, и критические температуры S1/F1/S2/F2 и S1/F1/N/F2 структур совпадают.

\section{4. Заключение}

Получены зависимости температуры сверхпроводящего перехода спинового вентиля S1/F1/S2/F2. Показана возможность немонотонного поведения $T_{\mathrm{c}}$ в такой структуре. В S1/F1/S2/F2 и S1/F1/N/F2 структуре возможно незначительное увеличение эффективности триплетного 
режима в сравнении с S1/F1/F2 структурой. Дополнительный слой небольшой толщины, необходимый для развязывания намагниченностей ферромагнитных слоев, увеличивает эффективность триплетного режима в сравнении с трехслойной структурой, тогда как для прямого режима дополнительный слой ее уменьшает. Это облегчает экспериментальное наблюдение триплетного спин-клапанного эффекта в гетероструктурах сверхпроводник-ферромагнетик, не использующих „экзотические“ ферромагнитные полуметаллы.

Для более детального и глубокого понимания механизмов реализации различных режимов спинового вентиля понадобится рассчитать распределение спинсинглетной и спин-триплетных компонент сверхпроводящего спаривания по слоям таких гетероструктур. Этому будет посвящена дальнейшая работа.

\section{Финансирование работы}

Работа поддержана проектом РНФ № 18-12-00459. В.Н.К. благодарит за поддержку проект ГПНИ Республики Беларусь „Физическое материаловедение, новые материалы и технологии“, подпрограмма „Нанотех“ (2016-2020).

\section{Конфликт интересов}

Авторы заявляют, что у них нет конфликта интересов.

\section{Список литературы}

[1] Ya.V. Fominov, A.A. Golubov, T.Yu. Karminskaya, M.Yu. Kupriyanov, R.G. Deminov, L.R. Tagirov. JETP Lett. 91, 308 (2010).

[2] R.R. Gaifullin, R.G. Deminov, L.R. Tagirov, M.Yu. Kupriyanov, Ya.V. Fominov, A.A. Golubov. submitted. Phys. Rev. B (2019). В печати.

[3] Ya.V. Fominov, A.A. Golubov, M.Yu. Kupriyanov. JETP Lett. 77, 510 (2003).

[4] L.R. Tagirov, M.Yu. Kupriyanov, V.N. Kushnir, A.S. Sidorenko. Superconducting triplet proximity and Josephson spin valves. In: „Functional nanostructures and metamaterials: from superconducting qubits to self-organized nanostructures". Springer Series: Nanoscience and Technology / Ed. A.S. Sidorenko, Springer International Publishing AG (2018). Ch. 2. P. 31-47.

[5] A. Singh, S. Voltan, K. Lahabi, J. Aarts. Phys. Rev. X 5, 021019 (2015).

[6] A.A. Kamashev, P.V. Leksin, N.N. Garifyanov, A.A. Validov, J. Schumann, V. Kataev, B. Büchner, I.A. Garifullin, J. Magn. Magn. Mater. 459, 7 (2018).

[7] F.S. Bergeret, A.F. Volkov, K.B. Efetov. Rev. Mod. Phys. 77, 1321 (2005).

[8] V.N. Kushnir. DSc dissertation. Minsk (2014).

[9] В.Н. Кушнир. Докл. БГУИР 3, 18 (2016).

[10] В.Н. Кушнир. Сверхпроводимость слоистиых структур. БНТУ, Минск (2010). 234 с.

Редактор Д.В. Жуманов 